Quim. Nova, Vol. 34, No. 2, 200-205, 2011

\title{
INFLUÊNCIA DAS CONDIÇÕES DE RESFRIAMENTO SOBRE AS PROPRIEDADES SUPERFICIAIS E ELETROQUÍMICAS DE ANODOS DIMENSIONALMENTE ESTÁVEIS
}

\author{
Élen C. G. Rufino* e Luiz A. de Faria \\ Instituto de Química, Universidade Federal de Uberlândia, 38400-902 Uberlândia - MG, Brasil \\ Leonardo M. da Silva \\ Departamento de Química, Universidade Federal dos Vales do Jequitinhonha e Mucuri, 39100-000 Diamantina - MG, Brasil
}

Recebido em 5/1/10; aceito em 21/8/10; publicado na web em 30/11/10

\begin{abstract}
INFLUENCE OF THE COOLING CONDITIONS ON SURFACE AND ELECTROCHEMICAL PROPERTIES OF DIMENSIONALLY STABLE ANODES. Surface and electrochemical properties of the dimensionally stable anode $\mathrm{Ti} /\left(\mathrm{Ru}_{0.3} \mathrm{Ti}_{0.7}\right)$ $\mathrm{O}_{2}$ were studied as a function of the annealing temperature using different conditions in order to perform the cooling process of the oxide films (conventional thermal shock and the slow cooling processes). It was found that surface and electrochemical properties for the oxygen evolution reaction are both affected through the cooling process, being the electrode prepared at $400{ }^{\circ} \mathrm{C}$ using the slow cooling process the less susceptible to wear. The Tafel slope obtained in the high overpotential domain was analysed in light of the apparent charge transfer coefficient.
\end{abstract}

Keywords: slow cooling treatment; morphology factor; apparent charge transfer coefficient.

\section{INTRODUÇÃO}

O sistema eletródico binário $\mathrm{Ti} /(\mathrm{Ru}+\mathrm{Ti}) \mathrm{O}_{2}$ constitui um dos principais tipos de anodos dimensionalmente estáveis, ADE. ${ }^{1-4}$ Além da produção de cloro, ${ }^{5}$ os ADE também podem ser utilizados em diferentes processos, como é o caso da remoção de poluentes orgânicos recalcitrantes presentes em efluentes aquosos. ${ }^{6}$

Diferentes métodos já foram propostos para o preparo de eletrodos do tipo ADE.$^{7-9}$ No entanto, se verifica que o método mais utilizado nos processos industriais é baseado no preparo de $\mathrm{ADE}$ policristalinos mediante a decomposição térmica da mistura dos sais precursores sobre o suporte metálico rugoso. ${ }^{1-4}$ Estudos revelam que a temperatura de decomposição (calcinação) da mistura precursora deve ser suficientemente alta para se obter a formação do filme de óxidos sem, no entanto, comprometer as propriedades mecânicas e elétricas do substrato metálico. ${ }^{1,10-13}$

Estudos revelam que a origem da influência do ADE sobre a velocidade dos processos eletródicos está relacionada com a presença de efeitos eletrocatalíticos verdadeiros (redução na barreira de ativação para o processo de transferência eletrônica) e aparentes, devida à influência da extensão da área eletroquimicamente ativa na eletrocatálise heterogênea. ${ }^{11-16}$

A influência da temperatura de calcinação sobre as propriedades superficiais e eletroquímicas de eletrodos do tipo ADE já foi relatada para diferentes sistemas binários e ternários. ${ }^{15-17}$ No entanto, se verifica nestes casos que o processo adotado na etapa de resfriamento dos filmes é o choque térmico baseado na remoção abrupta dos filmes do forno utilizado no processo de calcinação.

A influência do processo de resfriamento lento sobre as propriedades superficiais e eletrocatalíticas de sistemas binários $\left(\mathrm{IrO}_{2}+\mathrm{Ta}_{2} \mathrm{O}_{5}\right.$ e $\mathrm{IrO}_{2}+\mathrm{Nb}_{2} \mathrm{O}_{5}$ ) foi investigada com base nas reações de desprendimento de oxigênio e ozônio, ${ }^{14,18}$ onde se verificou que o processo de resfriamento lento pode modificar de forma considerável estas propriedades, bem como o tempo de vida útil dos eletrodos.

\footnotetext{
*e-mail: elencris85@yahoo.com.br
}

No presente artigo relata-se o estudo comparativo das propriedades superficiais e eletroquímicas de eletrodos do tipo ADE de composição nominal $\mathrm{Ti} /\left(\mathrm{Ru}_{0,3} \mathrm{Ti}_{0,7}\right) \mathrm{O}_{2}$ preparados por decomposição térmica em diferentes temperaturas, onde foram empregados os processos de resfriamento rápido (choque térmico) e lento.

\section{PARTE EXPERIMENTAL}

\section{Preparo dos eletrodos de trabalho}

Os filmes de composição nominal $\mathrm{Ti} /\left(\mathrm{Ru}_{0,3} \mathrm{Ti}_{07}\right) \mathrm{O}_{2}$ foram preparados por decomposição térmica ${ }^{19}$ a partir dos sais precursores $\left(\mathrm{RuCl}_{3}\right.$ e $\mathrm{TiCl}_{3}$ ) em diferentes temperaturas de calcinação (300, 400 e 500 $\left.{ }^{\circ} \mathrm{C}\right)$, mantendo-se a espessura nominal em $2 \mu \mathrm{m}$ e a área geométrica em $1,0 \mathrm{~cm}^{2}$. A montagem final dos eletrodos para a realização dos ensaios eletroquímicos foi finalizada conforme descrito na literatura. ${ }^{10,12}$ A etapa de resfriamento dos filmes após a calcinação de $1 \mathrm{~h}$ foi realizada empregando-se o "choque térmico", CT, onde os eletrodos foram retirados imediatamente da mufla resultando numa taxa de resfriamento dos filmes de $\sim 200{ }^{\circ} \mathrm{C} \mathrm{min}^{-1}$, e o resfriamento lento, RL, onde a mufla foi desligada e os eletrodos foram mantidos no seu interior até que a temperatura interna fosse igual à ambiente. Este procedimento resultou numa taxa média de resfriamento de 2 ${ }^{\circ} \mathrm{C} \min ^{-1}$. A aplicação da lei de Newton ${ }^{20}$ revelou que o processo de resfriamento lento dos filmes apresenta uma constante de troca de calor de $0,54 \mathrm{~h}^{-1}$, a qual é estritamente governada pelas características particulares da mufla utilizada.

\section{Caracterização dos filmes de óxidos}

$\mathrm{O}$ aspecto morfológico das superfícies dos filmes de óxidos e a composição química dos filmes foram investigados mediante estudos de microscopia eletrônica de varredura (MEV) e de espectroscopia dispersiva de Raios-X (EDX), os quais foram realizados empregandose um microscópio eletrônico da JEOL modelo T-300. 
A caracterização eletroquímica dos eletrodos foi efetuada mediante o registro de curvas voltamétricas, $E$ vs. $j$, a uma velocidade de varredura do potencial, $\mathrm{v}$, de $20 \mathrm{mV} \mathrm{s}{ }^{-1}$ em solução ácida $\left(\mathrm{H}_{2} \mathrm{SO}_{4} 0,5\right.$ mol $\mathrm{dm}^{-3}$ ), considerado-se o intervalo de potencial pseudocapacitivo de 0,2 a $1,4 \mathrm{~V}$ onde se localizam as transições redox superficiais no estado sólido do par $\mathrm{Ru}(\mathrm{III}) / \mathrm{Ru}(\mathrm{IV}){ }^{19}$

As curvas voltamétricas foram registradas $(1,025$ a 1,121 V) em função da velocidade de varredura do potencial $\left(5\right.$ a $\left.300 \mathrm{mV} \mathrm{s}^{-1}\right)$ com a finalidade de determinar a capacitância interfacial $(C)$ e o fator de morfologia $(\varphi)$ dos filmes. ${ }^{21}$

Todos os ensaios eletroquímicos foram efetuados empregando-se uma célula convencional de três compartimentos ${ }^{10,12}$ e o potencial do eletrodo de trabalho foi mensurado em relação ao eletrodo reversível de hidrogênio, ERH. A aquisição dos dados eletroquímicos ocorreu mediante o uso do potenciostato/galvanostato modelo PGSTAT20 da Autolab (Eco Chemie, Holanda).

\section{Estudo cinético da reação de desprendimento de oxigênio}

Curvas de polarização em condições quase-estacionárias $(0,5$ $\mathrm{mV} \mathrm{s}^{-1}$ ) foram registradas (duas varreduras cíclicas interruptas) a 25 ${ }^{\circ} \mathrm{C}$ em solução de $\mathrm{H}_{2} \mathrm{SO}_{4} 0,5 \mathrm{~mol} \mathrm{dm}^{-3}$, em condição de intenso borbulhamento com $\mathrm{N}_{2}$ ultrapuro. ${ }^{22} \mathrm{~A}$ ordem de reação referente ao $\mathrm{pH}$, $\varsigma(\mathrm{pH})$, foi determinada em potencial $(1,42 \mathrm{~V}$ vs. ERH) e força iônica $\left(1,0 \mathrm{~mol} \mathrm{dm}^{-3}\right)$ constantes, ${ }^{17,19}$ onde a partir da mistura apropriada de volumes das soluções estoques $\left(\mathrm{H}_{2} \mathrm{SO}_{4}\right.$ e $\left.\mathrm{Na}_{2} \mathrm{SO}_{4}\right)$ foram obtidos os valores desejados do $\mathrm{pH}$ cobrindo o intervalo de 0 a 1,5.

\section{Determinação do tempo de vida útil relativo dos eletrodos em condições aceleradas de desgaste}

$\mathrm{O}$ tempo de vida útil relativo dos eletrodos em meio ácido $\left(\mathrm{H}_{2} \mathrm{SO}_{4}\right.$ $0,5 \mathrm{~mol} \mathrm{dm}^{-3}$ ) foi avaliado a $25^{\circ} \mathrm{C}$ a partir de ensaios cronopotenciométricos, $E$ vs. $t$, aplicando-se uma densidade de corrente aparente de $0,9 \mathrm{~A} \mathrm{~cm}^{-2}$. Conforme descrito na literatura, ${ }^{13}$ o tempo de vida útil relativo dos eletrodos, $t_{6 \mathrm{~V}}$ foi estipulado arbitrariamente como sendo o tempo de eletrólise correspondente ao potencial do eletrodo de trabalho de 6,0 V (vs. ERH).

\section{RESULTADOS E DISCUSSÃO}

\section{Estudos de caracterização dos filmes de óxidos}

A Figura 1 mostra imagens das superfícies dos filmes de óxidos obtidas pela técnica MEV para o eletrodo $\mathrm{Ti} /\left(\mathrm{Ru}_{0,3} \mathrm{Ti}_{0,7}\right) \mathrm{O}_{2}$ preparado por decomposição térmica a $400{ }^{\circ} \mathrm{C}$ em diferentes condições de resfriamento (CT: choque térmico ou RL: resfriamento lento). Os dados referentes à composição percentual dos filmes são apresentados na Tabela 1.

A inspeção visual das diversas micrografias de MEV revelou que a morfologia dos filmes de composição nominal $\mathrm{Ti} /\left(\mathrm{Ru}_{0,3} \mathrm{Ti}_{0,7}\right)$ $\mathrm{O}_{2}$ é dependente da temperatura de calcinação e do procedimento de resfriamento. Em concordância com os dados obtidos para outros sistemas binários do tipo $\mathrm{ADE},{ }^{14,18}$ verificou-se (Figura 1) que a concentração superficial das rachaduras/fissuras é reduzida quando o filme de óxidos é resfriado lentamente.

O fato do processo de resfriamento lento originar filmes com morfologia superficial mais suave pode ser atribuído à troca de calor menos acentuada com o meio externo que, por sua vez, minimiza as tensões mecânicas estabelecidas na interface Ti/filme devidas às diferenças nos coeficientes de expansão térmica destes materiais. ${ }^{23}$

A análise da Tabela 1 revela que o tipo de resfriamento (CT ou RL) também influencia na composição percentual dos filmes,
(A)

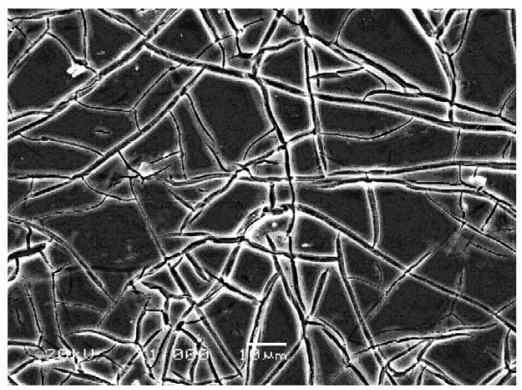

(B)

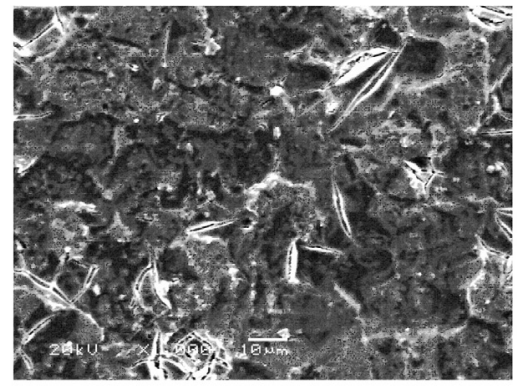

Figura 1. Imagens de microscopia eletrônica de varredura obtidas para o eletrodo de composição nominal $\left(R u_{0.3} T i_{0.7}\right) O_{2}$ preparado a $400^{\circ} \mathrm{C}$ : (a) $C T$ e (b) $R L$

Tabela 1. Dependência da composição percentual dos filmes obtida pela técnica EDX com a temperatura de calcinação e as condições de resfriamento

\begin{tabular}{cccc}
\hline $\mathrm{T} /{ }^{\circ} \mathrm{C}$ & Resfriamento & $\% \mathrm{TiO}_{2}$ & $\% \mathrm{RuO}_{2}$ \\
\hline 300 & $\mathrm{CT}$ & 69,3 & 30,7 \\
300 & $\mathrm{RL}$ & 75,9 & 24,1 \\
400 & $\mathrm{CT}$ & 69,2 & 30,8 \\
400 & $\mathrm{RL}$ & 75,8 & 24,2 \\
500 & $\mathrm{CT}$ & 70,7 & 29,3 \\
500 & $\mathrm{RL}$ & 78,9 & 21,1 \\
\hline
\end{tabular}

Composição nominal: $\mathrm{Ru}=30 \%$ e $\mathrm{Ti}=70 \%$.

ou seja, verifica-se que há uma discrepância considerável entre a composição verdadeira e a nominal (idealizada) para os eletrodos resfriados lentamente.

Devido à não volatilidade destes metais no intervalo de temperatura considerado, ${ }^{1,19}$ estes resultados, em princípio, indicariam a ocorrência de um processo de segregação superficial do Ti quando os filmes preparados em diferentes temperaturas são resfriados lentamente. No entanto, esta afirmação não pode ser empregada no presente estudo devido à energia de excitação utilizada na técnica EDX ser capaz de monitorar tanto a região superficial como as camadas de óxidos localizadas no cerne do filme.

O comportamento eletroquímico pseudocapacitivo típico do sistema eletródico $\mathrm{Ti} /\left(\mathrm{Ru}_{0,3} \mathrm{Ti}_{0,7}\right) \mathrm{O}_{2}$ em meio ácido é apresentado na Figura 2 , onde se verifica que a curva voltamétrica, $j$ vs. $E$, é caracterizada pela banda redox localizada em $\sim 0,60 \mathrm{~V}$ (vs. ERH), a qual é atribuída à transição redox superficial reversível no estado sólido $\mathrm{Ru}(\mathrm{III}) / \mathrm{Ru}(\mathrm{IV}){ }^{1,19}$

Estudos revelam que a carga voltamétrica total (pseudocapacitiva), $q^{*}$, obtida a partir da integração das curvas voltamétricas pode ser utilizada como medida relativa da área eletroquimicamente ativa dos eletrodos do tipo ADE. ${ }^{2,12,13,16,17}$ A Tabela 2 apresenta a dependência 


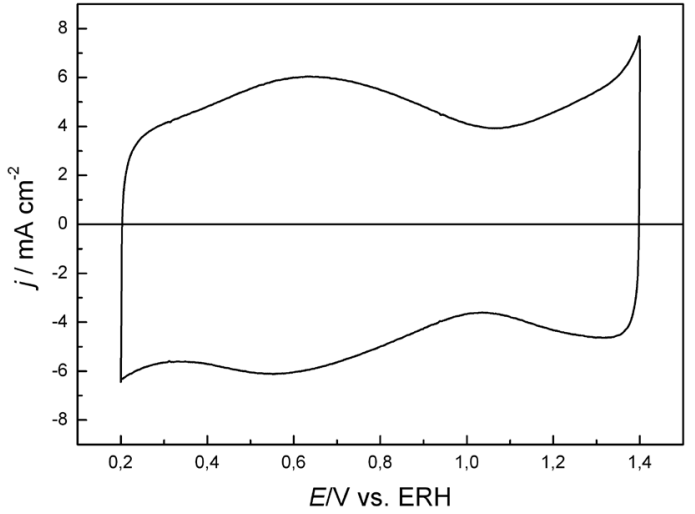

Figura 2. Voltamograma cíclico registrado no intervalo de potencial pseudocapacitivo a $20 \mathrm{mV} \mathrm{s}^{-1}$. Eletrodo: $\mathrm{Ti} /\left(\mathrm{Ru}_{0.3} \mathrm{Ti}_{0.7}\right) \mathrm{O}_{2}$. Eletrólito: $\mathrm{H}_{2} \mathrm{SO}_{4} 0,5 \mathrm{~mol}$ dm $m^{-3}$. Calcinação: $300^{\circ} \mathrm{C}$ e RL. T $=25^{\circ} \mathrm{C}$

de $q^{*}$ com o tipo de tratamento térmico utilizado. Conforme pode ser verificado, a análise comparativa dos valores de $q^{*}$ revela que a área eletroquimicamente ativa dos ADE é reduzida com o aumento da temperatura de calcinação, sendo este efeito mais pronunciado no caso do resfriamento lento.

Tabela 2. Influência da temperatura de calcinação e das condições de resfriamento dos filmes sobre a carga voltamétrica, $q^{*}$, obtida no intervalo pseudocapacitivo $(0,2$ a $1,4 \mathrm{~V}$ vs. ERH)

\begin{tabular}{ccc}
\hline $\mathrm{T} /{ }^{\circ} \mathrm{C}$ & $q^{*} / \mathrm{mC} \mathrm{cm}^{-2}$ \\
\hline & $\mathrm{CT}$ & $\mathrm{RL}$ \\
300 & 311 & 264 \\
400 & 71 & 62 \\
500 & 16 & 11 \\
\hline
\end{tabular}

$\mathrm{CT}=$ choque térmico; $\mathrm{RL}=$ resfriamento lento

Estes resultados são consistentes com o estudo de MEV, onde foi verificado que filmes apresentando uma menor concentração superficial de rachaduras/fissuras (redução da rugosidade superficial) são justamente aqueles que apresentam os menores valores de $q^{*}$.

Em virtude das peculiaridades dos ADE, ${ }^{2-4}$ verifica-se que o estudo de caracterização voltamétrica orientado pela análise comparativa dos parâmetros $q^{*}, C_{\mathrm{T}}$ (capacitância total) e $\varphi$ (fator de morfologia) constitui uma abordagem mais aprofundada acerca do sistema eletródico investigado. ${ }^{14,21,24,25}$ Portanto, perfis $j$ vs. $E$ no domínio pseudocapacitivo $(1,025-1,121 \mathrm{~V})$ foram registrados em função da velocidade de varredura do potencial, $v$, para os diferentes eletrodos.

Seguindo-se o tratamento proposto por Da Silva et al. ${ }^{21}$ os valores de corrente utilizados no cálculo da pseudocapacitância interfacial, $C=(d j / d v)_{\mathrm{E}}$, foram obtidos em potencial fixo $(1,11 \mathrm{~V})$ localizado próximo ao potencial de retorno anódico.

A Figura 3 mostra um perfil $j$ vs. $v$ característico de filmes com elevado grau de rugosidade..$^{14,24,25}$ Os valores das pseudocapacitâncias total, $C_{\mathrm{T}}$, e externa, $C_{\mathrm{E}}$, foram obtidos a partir dos coeficientes angulares observados nos domínios das baixas $\left(\nu<50 \mathrm{mV} \mathrm{s}^{-1}\right) \mathrm{e}$ elevadas $\left(v>70 \mathrm{mV} \mathrm{s}^{-1}\right)$ velocidades de varredura, respectivamente. A pseudocapacitância interna, $C_{\mathrm{I}}$, e o fator de morfologia, $\varphi$, foram calculados empregando-se as relações $C_{\mathrm{I}}=C_{\mathrm{T}}-C_{\mathrm{E}}$ e $\varphi=C_{\mathrm{I}} / C_{\mathrm{T}}$, respectivamente. ${ }^{21}$

A análise dos dados da Tabela 3 revela um aumento de $\varphi$ com a temperatura de calcinação. Verifica-se também que a influência mais pronunciada do processo de resfriamento (CT ou RL) sobre os parâmetros $C_{\mathrm{T}}$ e $\varphi$ ocorre para filmes preparados a $300^{\circ} \mathrm{C}$. Em con-

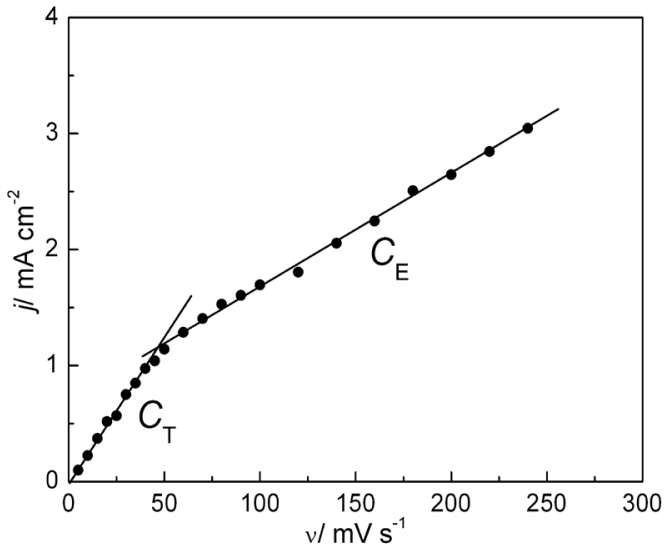

Figura 3. Dependência da densidade aparente de corrente voltamétrica, $j$, com a velocidade de varredura do potencial, $v$. Os valores de joram obtidos em 1,11 V (vs. ERH). Eletrólito: $\mathrm{H}_{2} \mathrm{SO}_{4} 0,5 \mathrm{~mol} \mathrm{dm} \mathrm{m}^{-3}$. Calcinação: $400{ }^{\circ} \mathrm{C} e$ CT. $T=25^{\circ} \mathrm{C}$

cordância com a literatura, ${ }^{26}$ verifica-se que os maiores valores de $\varphi$ (> $0,5)$ não são, necessariamente, acompanhados pelos maiores valores de $C_{\mathrm{T}}$. Este fato deve-se às diversas possibilidades de combinação das áreas mais internas e externas do filme rugoso que contribuem para o fator de morfologia..$^{21,26}$

Tabela 3. Dependência das pseudocapacitâncias externa, $C_{\mathrm{E}}$, interna, $C_{\mathrm{I}}$, e total, $C_{\mathrm{T}}$, e do fator de morfologia, $\varphi$, com a temperatura de calcinação e as condições de resfriamento dos filmes

\begin{tabular}{cccccc}
\hline $\mathrm{T} /{ }^{\circ} \mathrm{C}$ Resfriamento & $C_{\mathrm{E}}$ & $\begin{array}{c}C_{\mathrm{I}} \\
\mathrm{mF} \mathrm{cm}^{-2}\end{array}$ & $C_{\mathrm{T}}$ & $\varphi$ \\
\hline 300 & $\mathrm{CT}$ & 5,30 & 9,20 & 14,5 & 0,63 \\
300 & $\mathrm{RL}$ & 30,0 & 39,0 & 69,0 & 0,57 \\
400 & $\mathrm{CT}$ & 8,00 & 20,1 & 28,1 & 0,72 \\
400 & $\mathrm{RL}$ & 9,50 & 16,5 & 26,0 & 0,63 \\
500 & $\mathrm{CT}$ & 3,10 & 18,0 & 21,3 & 0,85 \\
500 & $\mathrm{RL}$ & 3,10 & 16,6 & 19,7 & 0,84 \\
\hline
\end{tabular}

$\mathrm{CT}=$ choque térmico; $\mathrm{RL}=$ resfriamento lento

\section{Estudo cinético da reação de desprendimento de oxigênio em meio ácido}

A Figura 4 mostra uma curva de Tafel antes e após a correção para a queda ôhmica $\left(j R_{\Omega}\right) \cdot{ }^{19,26}$ Os coeficientes de Tafel obtidos nos domínios dos baixos, $b_{1}$, e elevados, $b_{2}$, sobrepotenciais em função das condições de tratamento térmico são apresentados na Tabela 4 , onde se verifica que os valores de $b$, principalmente $b_{1}$, estão em boa concordância com os dados relatados por diferentes autores para a RDO em meio ácido empregando-se o sistema eletródico Ti/ $\left(\mathrm{Ru}_{0,3} \mathrm{Ti}_{0,7}\right) \mathrm{O}_{2} \cdot{ }^{1,16,19}$

Estudos revelam que variações no coeficiente de Tafel para a RDO com o aumento do sobrepotencial podem estar associadas a mudanças na etapa determinante da velocidade do processo eletródico e/ou a variações ocorridas no coeficiente de transferência de carga aparente, $\alpha_{\text {apa }}$, devidas à não idealidade do processo de transferência de carga para os eletrodos sólidos na condição de forte polarização. ${ }^{17,22,24,26,27}$

A análise dos dados da Tabela 4 revela que os valores de $b_{1}$ obtidos para os eletrodos mais compactos são pouco superiores $(\sim 10 \mathrm{mV})$ quando comparados àqueles obtidos para eletrodos menos compactos (Tabelas 2 e 3). Já no caso do coeficiente $b_{2}$, verifica-se que o aumento 


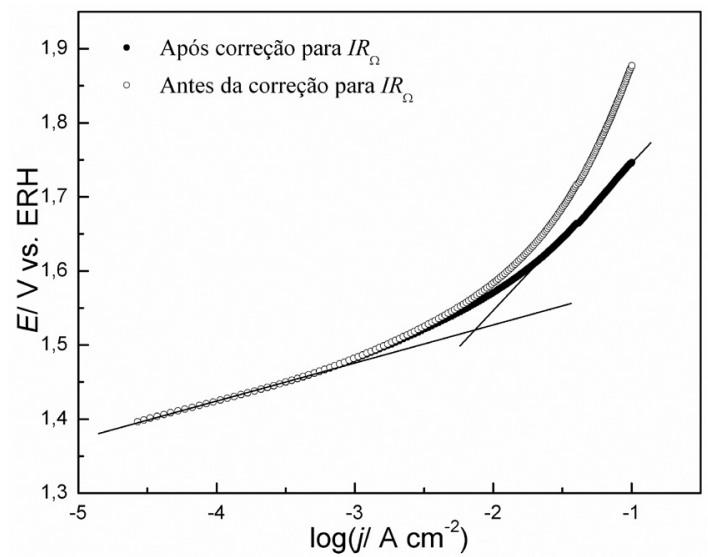

Figura 4. Curva de Tafel referente à reação de desprendimento de oxigênio, RDO. Solução: $\mathrm{H}_{2} \mathrm{SO}_{4} 0,5 \mathrm{~mol} \mathrm{dm} \mathrm{m}^{-3}$. Calcinação: $400^{\circ} \mathrm{C}$ e CT. T $=25^{\circ} \mathrm{C}$

Tabela 4. Dependência do coeficiente de Tafel obtido para a RDO no domínio dos baixos, $b_{1}$, e elevados, $b_{2}$, sobrepotenciais com a temperatura de calcinação e as condições de resfriamento dos filmes

\begin{tabular}{cccccc}
\hline \multicolumn{2}{c}{$\mathrm{T} /{ }^{\circ} \mathrm{C}$ Resfriamento } & $b_{1} / \mathrm{mV}$ & $b_{2} / \mathrm{mV}$ & $\alpha_{\text {apa }}{ }^{\mathrm{I}}$ & $\alpha_{\text {apa }}{ }^{\mathrm{II}}$ \\
\hline 300 & $\mathrm{CT}$ & 44 & 88 & 0,67 & 0,34 \\
300 & $\mathrm{RL}$ & 42 & --- & --- & 0,34 \\
400 & $\mathrm{CT}$ & 50 & 214 & 0,28 & 0,18 \\
400 & $\mathrm{RL}$ & 54 & 211 & 0,28 & 0,10 \\
500 & $\mathrm{CT}$ & 54 & 220 & 0,27 & 0,10 \\
500 & $\mathrm{RL}$ & 52 & 296 & 0,20 & 0,14 \\
\hline
\end{tabular}

considerável da inclinação de Tafel com a temperatura de calcinação (RL e CT) é acompanhado pelo aumento de $\varphi$ (Tabela 3 ) e o decréscimo de $q^{*}$ (Tabela 2). Diversos estudos revelam que o parâmetro $b$ ou, mais precisamente, o $\alpha_{\text {apa }}$ pode ser influenciado pela morfologia do eletrodo devido à influência da distribuição não uniforme do campo elétrico superficial associada com a flutuação espacial da constante dielétrica no plano de Helmholtz da dupla camada elétrica sobre o processo de transferência de carga. ${ }^{22,26,33,37}$

A determinação da ordem de reação foi efetuada em sobrepotencial constante, $\varsigma(\mathrm{pH})_{\eta}$, no domínio dos baixos sobrepotenciais onde, de acordo com a literatura, ${ }^{16,17,19}$ há normalmente uma dependência da velocidade da reação, $j$, com o $\mathrm{pH}$ devida à segunda etapa de transferência eletrônica do mecanismo eletródico ser a determinante da velocidade. ${ }^{17}$

Verificou-se para os eletrodos preparados em diferentes condições de calcinação que os coeficientes angulares obtidos a partir dos perfis lineares $\log \left(j_{\text {RDO }}\right)$ vs. $\log \left(\left[\mathrm{H}^{+}\right]\right)$são nulos, ou seja, $\varsigma(\mathrm{pH})_{\eta}=0$. No entanto, visto que o eletrodo de referência utilizado foi o ERH, tem-se que a ordem de reação obtida neste caso é aparente devido ao potencial do ERH variar linearmente com o $\mathrm{pH}$. De acordo com a literatura, ${ }^{17,19}$ somente a ordem da reação determinada em potencial constante, $\varsigma(\mathrm{pH})_{\mathrm{E}}$, é quimicamente significativa (real).

A ordem da reação real ${ }^{19}$ referente ao $\mathrm{pH}$ foi obtida empregandose a seguinte relação:

$$
\varsigma(\mathrm{pH})_{\mathrm{E}}=\varsigma(\mathrm{pH})_{\eta}+\gamma
$$

onde $\gamma$ é o parâmetro na inclinação de Tafel. Para o caso da equação cinética ser caracterizada por um coeficiente de Tafel de 2,303RT/(1 $+\alpha_{\text {apa }}$, obtém-se a identidade $\gamma=1+\alpha_{\text {apa }}$.

Portanto, visto que $\varsigma(\mathrm{pH})_{\eta}=0$ conclui-se que $\varsigma(\mathrm{pH})_{\mathrm{E}}=\gamma$, ou seja, a ordem da reação é dependente do coeficiente de Tafel. ${ }^{17,19}$
Com base nos dados cinéticos experimentais e nas ponderações apresentadas, pode-se propor que a RDO pode ser descrita pelo seguinte mecanismo eletródico: ${ }^{19}$

$$
\begin{gathered}
\equiv \mathrm{S}+\mathrm{H}_{2} \mathrm{O} \rightarrow \equiv \mathrm{S}-\mathrm{OH}^{\bullet}+\mathrm{H}^{+}+\mathrm{e} \\
\equiv \mathrm{S}-\mathrm{OH}^{\bullet} \rightarrow \equiv \mathrm{S}-\mathrm{O}^{\bullet}+\mathrm{H}^{+}+\mathrm{e} \\
\equiv \mathrm{S}-\mathrm{O}^{\bullet}+\equiv \mathrm{S}-\mathrm{O}^{\bullet} \rightarrow 2 \equiv \mathrm{S}+\mathrm{O}_{2}
\end{gathered}
$$

onde $\equiv \mathrm{S}$ é um sítio superficial ativo.

Aplicando-se o tratamento cinético proposto na literatura ${ }^{28,29}$ para os processos eletródicos irreversíveis em multietapas, obtém-se a expressão para a dependência da corrente com o sobrepotencial para o caso da etapa I ser a determinante da velocidade:

$j_{I}=4 F A k^{o}\left\{\left(1-\theta_{\text {OH }}\right) \exp \left[\alpha_{\text {apa }}^{\mathrm{I}} F \eta / R T\right]-\theta_{\text {OH }} \exp \left[-\left(1-\alpha_{\text {apa }}^{\mathrm{I}}\right) F \eta / R T\right]\right\}$

onde $k^{\circ}$ é a constante cinética independente do potencial, $\theta_{\mathrm{OH}}$ é a cobertura superficial do radical hidroxila, $\eta\left(=E-E_{\text {eq. }}\right)$ é o sobrepotencial, e $\alpha_{\text {apa }}^{\mathrm{I}}$ é o coeficiente de transferência eletrônica aparente ${ }^{26}$ referente à etapa I (descarga primária da água).

Em condição de alto-campo $(\eta>0,1 \mathrm{~V})$ e assumindo-se que (1 - $\left.\theta_{\mathrm{OH}}\right) \cong 1,{ }^{28,29}$ obtém-se a seguinte expressão cinética para o caso da etapa I ser a determinante da velocidade: ${ }^{29}$

$$
j_{I}=4 F A k^{o} \exp \left[\alpha_{\text {apa }}^{\mathrm{I}} F \eta / R T\right]
$$

que prevê uma ordem de reação nula com respeito ao $\mathrm{pH}$.

A expressão cinética para o caso da etapa II ser a determinante da velocidade pode ser obtida considerando-se a etapa I em condição de pré-equilíbrio, de onde se obtém a seguinte relação: ${ }^{17}$

$$
j_{I I}=4 F A k^{o}\left[\mathrm{H}^{+}\right]^{-1} \exp \left[\left(1+\alpha_{\text {apa }}^{\text {II }}\right) F \eta / R T\right]
$$

onde $\alpha_{\text {apa }}^{\mathrm{I}}$ é o coeficiente de transferência eletrônica aparente ${ }^{26}$ referente à etapa II (segunda transferência de carga).

De acordo com a literatura, ${ }^{17}$ a Equação 4 deve ser corrigida para a influência do pH, considerando-se o equilíbrio ácido-base interfacial, descrito pela seguinte relação:

$$
\equiv \mathrm{S}-\mathrm{OH} \rightleftharpoons \equiv \mathrm{S}-\mathrm{O}^{-}+\mathrm{H}^{+}
$$

Assumindo-se comportamento reversível para o equilíbrio descrito pela Equação 5, obtém-se a seguinte equação cinética: ${ }^{17}$

$$
j_{I I}=4 F A k_{\text {apa }}\left[\mathrm{H}^{+}\right]^{-\left(1+\alpha_{\text {apa }}\right)} \exp \left[\left(1+\alpha_{\text {apa }}^{\text {II }}\right) F \eta / R T\right]
$$

A análise da Equação 6 revela que $\gamma=1+\alpha^{\mathrm{II}}{ }_{\text {apa }}$. Portanto, considerando-se $\alpha_{\text {apa }} \cong 0,5$ obtém-se $\varsigma(\mathrm{pH})_{\mathrm{E}} \cong 1,5$, o qual é condizente com diversos dados da literatura. ${ }^{17,19,30} \mathrm{O}$ fato da ordem de reação com respeito ao $\mathrm{pH}$ para a reação de desprendimento de oxigênio não ser influenciada pela temperatura de calcinação quando o parâmetro $\alpha$ é considerado uma constante $(=0,5)$ está de acordo com alguns relatos da a literatura. ${ }^{17}$

No entanto, na presente abordagem considerando-se o $\alpha^{\mathrm{II}}{ }_{\text {apa }}$ (Tabela 4) foi verificado que a ordem de reação varia no intervalo de 1,10 a 1,34, dependendo do tipo de tratamento térmico utilizado.

Com base nas Equações 3 e 6 e considerando-se a definição $b$ $(\partial E / \partial \log (j))_{T}$, obtém-se os coeficientes de Tafel teóricos para a etapa de descarga primária da água $\left(b^{\mathrm{I}}=2,303 R T / \alpha_{\text {apa }}^{\mathrm{I}} F\right)$ e da segunda transferência de carga $\left(b^{\Pi \mathrm{I}}=2,303 R T /\left(1+\alpha_{\text {apa }}^{\mathrm{I}}\right) F\right)$, respectivamente. 
Para o caso idealizado envolvendo a transferência de carga $(\alpha=0,5)$ a $25{ }^{\circ} \mathrm{C}$, verifica-se que coeficientes de Tafel de 120 e $40 \mathrm{mV}$ são previstos para casos onde as etapas I e II sejam as determinantes da velocidade, respectivamente.

Comparando-se os valores teóricos previstos para $b^{\mathrm{I}}$ e $b^{\mathrm{II}}$ com os dados experimentais da Tabela 4 , verifica-se que $\alpha_{\text {apa }} \neq 0,5$ para a maioria dos casos. Os maiores desvios da idealidade foram verificados para o domínio dos elevados sobrepotenciais onde se constatou que $b_{2} \gg 120 \mathrm{mV}$ para os eletrodos preparados a 400 e $500{ }^{\circ} \mathrm{C}$.

A revisão da literatura revelou que valores de $\alpha_{\text {apa }} \neq 0,5$ são, de fato, obtidos para diversos processos eletródicos envolvendo eletrodos sólidos. ${ }^{17,22,24,26,27}$ Conforme pode ser constatado na monografia de Erdey-Grúz ${ }^{28}$ e em outros trabalhos, ${ }^{31-33}$ a "incorporação" original do parâmetro $\alpha$ na equação referente à cinética eletródica foi fundamentada em várias idealizações concernentes à transferência de carga na interface eletrodo/solução.

Várias expressões teóricas já foram propostas visando representar o coeficiente de transferência de carga aparente..$^{27,31-34}$ No entanto, a revisão da literatura revela que ainda não foi proposto um modelo unificado referente ao processo de transferência de carga em eletrodos sólidos que incorpore a influência dos diferentes parâmetros majoritários pertinentes à interface eletrodo/solução.

Modelos quânticos para o processo de transferência de carga na interface eletrodo/solução já foram discutidos na literatura ${ }^{35-37}$ tendo como base, em alguns casos, a reação de desprendimento de hidrogênio como processo eletródico modelo. No entanto, verifica-se que a aplicação adequada deste tipo de modelo a processos multieletrônicos complexos, como o da RDO, constitui uma tarefa bastante difícil.

Conforme relatado anteriormente, ${ }^{22,26,38}$ torna-se oportuno o uso do parâmetro $\alpha_{\text {apa }}$ na interpretação dos dados cinéticos referentes a processos eletródicos complexos. Neste contexto, a racionalização dos dados cinéticos experimentais frente ao mecanismo eletródico proposto permite inferir que a segunda etapa de transferência eletrônica, que envolve o rompimento da ligação no complexo superficial ativo $\equiv \mathrm{S}-\mathrm{OH}^{\bullet}$ resultando na formação do oxigênio atômico adsorvido, constitui a etapa lenta do processo no domínio dos baixos sobrepotenciais (Equação 6).

$\mathrm{O}$ fato do parâmetro $\alpha^{\mathrm{II}}{ }_{\text {apa }}$ sofrer ligeira tendência de decréscimo com a elevação da temperatura de calcinação (RL e CT) não pode, em princípio, ser correlacionado com a redução da concentração superficial dos sítios ativos (Tabela 2), visto que foram obtidos valores de $b_{1}$ (RL e CT) pouco discrepantes.

No entanto, considerando-se os dados da Tabela 3 , verifica-se que a redução do $\alpha_{\text {apa }}^{\text {II }}$ é acompanhada pelo aumento expressivo do fator de morfologia dos filmes, $\varphi$. Conforme discutido anteriormente,,$^{22,26}$ este tipo de constatação fornece indícios de que efeitos de natureza morfológica, como é o caso da dispersão não uniforme do campo elétrico superficial, podem influenciar o processo de transferência eletrônica reduzindo, portanto, a fração efetiva do potencial elétrico no plano de aproximação máxima da dupla camada elétrica que contribui para o processo de transferência de carga. ${ }^{27,32,33}$

Os dados cinéticos obtidos para o domínio dos elevados sobrepotenciais indicam que a oxidação da água envolvendo a formação do radical hidroxila (descarga primária da água) constitui a etapa determinante do processo eletródico. Verifica-se neste caso a redução do $\alpha_{\text {apa }}^{\mathrm{I}}$, acompanhada pelo aumento de $\varphi$, com o aumento da temperatura de calcinação (RL e CT) sendo, no entanto, esta redução mais pronunciada quando comparada aos dados obtidos no domínio dos baixos sobrepotenciais.

Conforme mostrado na Tabela 4, na grande maioria dos casos, os parâmetros $\alpha_{\text {apa }}^{\mathrm{I}}$ e $\alpha^{\mathrm{II}}{ }_{\text {apa }}$ diminuem com o aumento da temperatura de calcinação. Além disso, foi constatado neste estudo que o parâmetro morfológico $\varphi$ (Tabela 3) aumenta com a temperatura de calcinação. Portanto, considerando-se que algumas das especulações teóricas a respeito dos desvios da idealidade para o processo de transferência de carga em eletrodos sólidos contemplam o fenômeno da dispersão do campo elétrico superficial induzido pela rugosidade superficial, ${ }^{14,22,26}$ pode-se inferir que o decréscimo de $\alpha_{\text {apa }}$ verificado com o aumento da temperatura de calcinação é ocasionado pelo aumento da dispersão do campo elétrico superficial, o qual pode ser vislumbrado indiretamente no presente estudo pelo parâmetro morfológico $\varphi$.

\section{Determinação do tempo de vida útil relativo dos ADE em condições aceleradas de desgaste}

A Figura 5 mostra um perfil cronopotenciométrico ( $E$ vs. $t$ ) para a RDO em $\mathrm{H}_{2} \mathrm{SO}_{4} 0,5 \mathrm{~mol} \mathrm{dm}^{-3}$ durante a aplicação de uma densidade de corrente aparente de $0,9 \mathrm{~A} \mathrm{~cm}^{-2}$ a $25^{\circ} \mathrm{C}$, enquanto que a Figura 6 mostra a dependência do tempo de vida útil relativo, $t_{6 \mathrm{~V}}$, com a temperatura de calcinação (RL e CT).

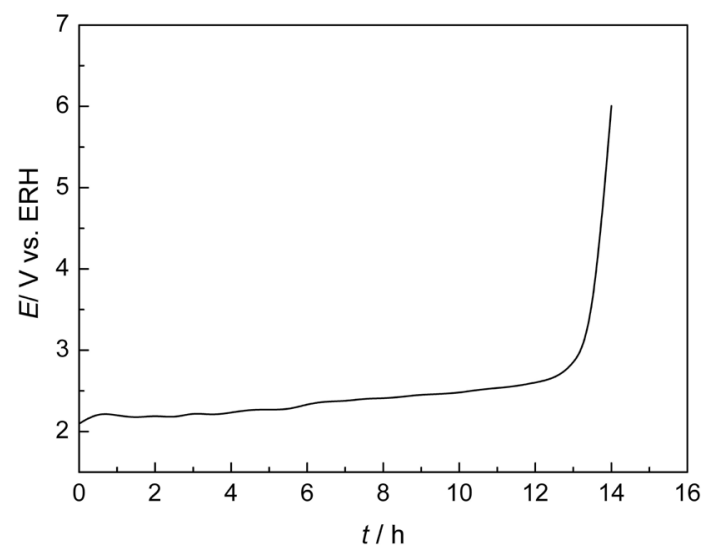

Figura 5. Curva cronopotenciométrica referente à RDO em condições de desgaste acelerado do eletrodo: $j=0,9 \mathrm{~A} \mathrm{~cm}^{-2}$ e $T=25 \pm 1{ }^{\circ} \mathrm{C}$. Eletrólito: $\mathrm{H}_{2} \mathrm{SO}_{4} 0,5 \mathrm{~mol} \mathrm{dm} \mathrm{m}^{-3}$. Calcinação: $400^{\circ} \mathrm{C}$ e RL. $\mathrm{T}=25^{\circ} \mathrm{C}$

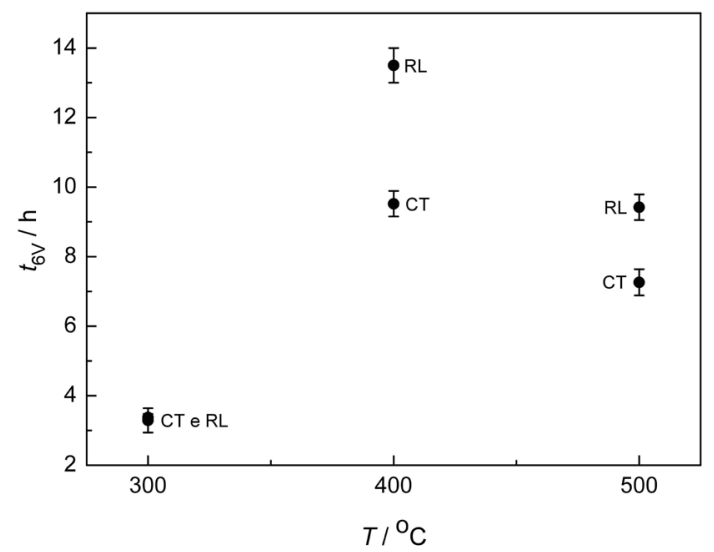

Figura 6. Dependência do tempo de vida útil relativo dos eletrodos, $t_{6 \mathrm{v}}$ com a temperatura de calcinação e com o tipo de resfriamento (CT e RL). Condições: $j=0,9 \mathrm{~A} \mathrm{~cm}^{-2}$ e $\mathrm{T}=25^{\circ} \mathrm{C}$. Eletrólito: $\mathrm{H}_{2} \mathrm{SO}_{4} 0,5 \mathrm{~mol} \mathrm{dm}^{-3}$

A análise da Figura 5, cujo aspecto qualitativo é representativo dos demais casos investigados, revela que o potencial do anodo, $E$, permanece praticamente constante até $\sim 4$ h de intensa eletrólise (condição de estabilidade), enquanto que para o intervalo de $\sim 4$ a 12 h é verificado um aumento linear de $E$, o qual denuncia a degradação e/ou passivação do ADE devido ao crescimento gradativo da intercamada de $\mathrm{TiO}_{x}$ na interface $\mathrm{Ti} / \mathrm{filme} .{ }^{13} \mathrm{O}$ processo de desativação do ADE foi verificado em $t>12 \mathrm{~h}$ mediante o aumento abrupto de $E$. 
Este tipo de comportamento está de acordo com a literatura, ${ }^{13}$ onde se empregando diferentes técnicas eletroquímicas no estudo da desativação de eletrodos do tipo ADE foram propostos critérios para a análise dos perfis $E$ vs. $t$ tendo como base o parâmetro denominado "fator de instabilidade - $\Lambda$ " e a "função de transferência temporal" representativa da dinâmica de desgaste do eletrodo.

A análise da Figura 6 permite inferir que a desativação dos ADE de composição nominal $\mathrm{Ti} /\left(\mathrm{Ru}_{0,3} \mathrm{Ti}_{0,7}\right) \mathrm{O}_{2}$, preparados em diferentes condições de calcinação, é principalmente caracterizada por dois aspectos: o tempo de vida útil relativo é máximo para o ADE preparado a $400{ }^{\circ} \mathrm{C}$ (CT e RL) e, o uso do processo de resfriamento lento acarreta em ganhos no tempo de vida útil relativo somente para ADE preparados em temperaturas mais elevadas $\left(400\right.$ e $\left.500{ }^{\circ} \mathrm{C}\right)$.

O fato dos eletrodos preparados a 400 e $500{ }^{\circ} \mathrm{C}$ e submetidos ao processo de resfriamento lento apresentarem maiores valores de $t_{6 \mathrm{~V}}$, quando comparados aos eletrodos resfriados em condição de choque térmico, pode ser atribuído à presença de uma menor concentração superficial de rachaduras/fissuras profundas. ${ }^{8,13}$

Neste contexto, à medida que os filmes de óxidos se tornam mais compactos, ocorre que a penetração do eletrólito nas rachaduras/ fissuras até a superfície do suporte de titânio se torna menos provável impedindo, portanto, a passivação gradativa da interface Ti/filme. ${ }^{13}$ Aliada a isto, a erosão mecânica provocada pelo intenso desprendimento de gás no interior destes defeitos superficiais se torna menos pronunciada, aumentando o tempo de vida útil.

\section{CONCLUSÕES}

O estudo de caracterização empregando-se técnicas ex-situ e insitu revelou que a morfologia dos ADE de composição nominal Ti/ $\left(\mathrm{Ru}_{0,3} \mathrm{Ti}_{0,7}\right) \mathrm{O}_{2}$ é dependente do tipo de resfriamento adotado no processo de calcinação (choque térmico ou lento), sendo a concentração superficial das rachaduras/fissuras minimizada mediante o uso do processo de resfriamento lento. Foi também verificado o aumento do fator de morfologia $(\varphi)$ dos filmes com a temperatura de calcinação.

O estudo cinético da reação de desprendimento de oxigênio revelou um aumento considerável da inclinação de Tafel presente no domínio dos elevados sobrepotenciais com a temperatura de calcinação para os diferentes processos de resfriamento empregados. Foi verificada, para os dados cinéticos obtidos no domínio dos baixos sobrepotenciais, uma variação da ordem de reação fracionária com o aumento da temperatura de calcinação.

Os valores do coeficiente de Tafel e as ordens de reação fracionárias foram interpretados no presente estudo do ponto de vista teórico mediante a dependência do coeficiente de transferência de carga aparente, $\alpha_{\text {apa }}$, com o aumento do fator de morfologia (dispersão do campo elétrico superficial).

O estudo cronopotenciométrico conduzido em função das condições de calcinação dos $\mathrm{ADE}$ revelou que o tempo de vida útil é máximo para o eletrodo preparado a $400{ }^{\circ} \mathrm{C}$. Foi também verificado que o uso do processo de resfriamento lento resulta em ganhos no tempo de vida útil relativo somente para eletrodos preparados em temperaturas mais elevadas $\left(400\right.$ e $\left.500{ }^{\circ} \mathrm{C}\right)$.

\section{AGRADECIMENTOS}

Ao $\mathrm{CNPq}$ pela bolsa concedida e à FAPEMIG pelo apoio financeiro.

\section{REFERÊNCIAS}

1. Trasatti, S.; Electrodes of Conductive Metallic Oxides, Elsevier: Amsterdam, 1980 .
2. Trasatti, S.; Electrochim. Acta 1991, 36, 225.

3. Trasatti, S.; Electrochim. Acta 2000, 45, 2377.

4. Guerrini, E.; Trasatti, S.; Russ. J. Electrochem. 2006, 42, 1017.

5. Trasatti, S.; Electrochim. Acta 1987, 32, 369.

6. Comninellis, C. Em Environmental Oriented Electrochemistry; Sequeira, C. A. C., ed.; Elsevier: Amsterdam, 1994, vol. 59.

7. Panic, V. V.; Nikolic, B. Z.; J. Serb. Chem. Soc. 2008, 73, 1083.

8. Pelegrino, R. R. L.; Vicentin, L. C.; Andrade, A. R.; Bertazzoli, R.; Electrochem. Commun. 2002, 4, 139.

9. Gandet, J.; Tavares, A. C.; Trasatti, S.; Guay, D.; Chem. Mater. 2005, 17, 1570.

10. Da Silva, L. M.; De Faria, L. A.; Boodts, J. F. C.; Electrochim. Acta 2000, 45, 2719.

11. Santana, M. H. P.; Da Silva, L. M.; De Faria, L. A.; Eletrochim. Acta 2003, 48, 1885.

12. De Faria, L. A.; Boodts, J. F. C.; Trasatti, S.; Electrochim. Acta 1992 , 37, 2511.

13. Da Silva, L. M.; Fernandes, K. C.; De Faria, L. A.; Boodts, J. F. C.; Electrochim. Acta 2004, 49, 4893.

14. Da Silva, L. M.; Franco, D. V.; De Faria, L. A.; Boodts, J. F. C.; Electrochim. Acta 2004, 49, 3977.

15. Burke, L. D.; Murphy, O. J.; J. Electrochem. Soc. 1979, 96, 19.

16. Burke, L. D.; Murphy, O. J.; O’Neill, J. F.; Faraday Trans. 1977, 73, 1659.

17. Angelineta, C.; Falciola, M.; Trasatti, S.; J. Electroanal. Chem. 1986, 205, 347.

18. Santana, M. H. P.; De Faria, L. A.; Boodts, J. F. C.; J. Appl. Electrochem. 2005, 35, 915 .

19. Boodts, J. F. C.; Trasatti, S.; J. Electrochem. Soc. 1990, 137, 3784.

20. Dence, J. B.; Mathematical Techniques in Chemistry, Wiley: New York, 1975.

21. Da Silva, L. M.; De Faria, L. A.; Boodts, J. F. C.; Electrochim. Acta 2001, 47, 395 .

22. Da Silva, L. M.; De Faria, L. A.; Boodts, J. F. C.; Electrochim. Acta 2003, 48, 699 .

23. Kameyama, K.; Tsukada, K.; Yahikozawa, K.; Takasu, Y.; J. Electrochem. Soc. 1993, 140, 966.

24. Merrill, M. D.; Dougherty, R. C.; J. Phys. Chem. C 2008, 112, 3655.

25. Malpass, G. R. P.; Neves, R. S.; Rodrigo, S.; Motheo, A. J.; Electrochim. Acta 2006, 52, 936.

26. Fernandes, K. C.; Da Silva, L. M.; Boodts, J. F. C.; De Faria, L. A.; Electrochim. Acta 2006, 51, 2809.

27. Lefebvre, M. C. Em Modern Aspects of Electrochemistry; Conway, B. E.; Bockris, J. O'M.; White, R. E., eds.; Kluwer: New York, 2002, vol. 32.

28. Erdey-Grúz, T.; Kinetics of Electrode Processes, Wiley: New York, 1972.

29. Böckris, J. O'M.; Reddy, A. K. N.; Gamboa-Aldeco, M. E.; Modern Electrochemistry, 2A: Fundamentals of Electrodics, $2^{\text {nd }}$ ed., Kluwer: New York, 2000.

30. Da Silva, L. M.; De Faria, L. A.; Boodts, J. F. C.; Electrochim. Acta 2001, 46, 1369.

31. Mayer, R. E.; J. Electrochem. Soc. 1960, 107, 847.

32. Gileadi, E.; Kirowa-Eisner, E.; Corros. Sci. 2005, 47, 3068.

33. Brenet, J.; Traore, K.; Transfer Coefficients in Electrochemical Kinetics, Academic Press: London, 1971.

34. Kriksunov, L. B.; Élektrokhimyia 1992, 28, 792.

35. Dogonadze, R. R.; Kuznetsov, A. M.; Levich, V. G.; Electrochim. Acta 1968, 13, 1025.

36. Tsirlina, G. A.; Kuznetsov, A. M.; Petrii, O. A.; Kharkats, Yu. I.; Élektrokhimyia 1999, 35, 938.

37. Böckris, J. O'M.; Khan, S. U. M.; Quantum Electrochemistry, Plenum Press: New York, 1979.

38. Franco, D. V.; Da Silva, L. M.; Jardim. W. F.; Boodts, J. F. C.; J. Braz. Chem. Soc. 2006, 17, 746. 